\title{
Calculation of Cross Section area and Mean Free path for Interaction of Neutrons with Mammalians cell of Chinese hamster cells (V79)
}

Received : 7/12/2017

Accepted : 18/1/2018

\author{
Hassan M. Jaber Al-Ta'ii \\ University of Muthanna - College of Science- Department of Physics \\ Email: hassankirkukly@gmail.com; hassankirkukly@mu.edu.iq
}

\begin{abstract}
$\underline{\text { Abstract: }}$
This paper focused on finding the cross-section area and mean free path of the irradiated mammalians cell for the Chinese hamster cells (V79). They were computed based on two models namely Linear Quadratic model (LQ) and Repairable Conditionally Repairable model (RCR). The energy influence on the cross-section area and mean free path was studied. The calculated mean free path by LQ model was higher than the value that obtained by RCR model. Furthermore, the mean free path value is reduced with increasing the linear energy transfer for both models. However, the cross-section area showed a different trend with augmenting the linear energy transfer.
\end{abstract}

Keywords: Neutrons; Free Path; Cross section; LET.

\section{Introduction:}

The cell inactivation was studied under the ionizing radiation through many methods such as inactivation cross section, survival curves, The Relative Biological Effectiveness (RBE) and dose rate survival requirement. Watt and his group paved to study the cross section under the radiation impact and radiation kinds, which were worked for obtained the general behavior of radiation impact on the target [1]. Neutron is a sole particle, have the properties such as no electric charge that means the electromagnetic wave does not impact on it. It has a relatively large mass, subject to radioactive decay. The cosmic rays are consider the main source for the free neutrons in the nature [2]. Neutrons are much more essential than any other radiation particles due to it is a neutral particle, does not have the capability for direct ionization of matter. The elastic collision consider the main mechanism of neutron by it interaction with the matter [2, $3]$. The radiation beam when the incident on the matter, some of these radiations capable absorbed totally, some of these might be scattered and some of these may pass straight through without any interaction at all. The ionization process impact, in the molecules of living cells attributed to the radiation. These ionization products were obtained from removing electrons from atoms, forming ions or charged atoms. The ions can be reacted with other atoms in the cell thereby generated the damage. The cross section can be defined as the probability that the specified event will occur per incident particle per target center per unit area. The Mean Free Path (MFP) can define the average distance between the collisions in the target. The MFP relies on the diameter of the target particle, number of target particles per unit volume and the energy $[4,5]$.

Azooz and co-worker were obtained to the higher inactivation cross section of V79 from the incident proton when the mean free path is 
range (2.0-5.0 nm) [6]. Azooz and Meerkhan 2009, study the inactivation of V79 cells and CHO-K1 cells were irradiated with ${ }^{12} \mathrm{C}$ ions, protons, ${ }^{3} \mathrm{He}$ ions and neutrons, which observed the mean free path $\lambda$ were growing and with decline inactivation cross section $\sigma$ generally; this reduce was followed an exponential form in the inactivation of V79 cells by ${ }^{12} \mathrm{C}$ ions and $\mathrm{CHO}-\mathrm{K} 1$ cells by ${ }^{3} \mathrm{He}$ ions [7]. Furthermore, our study in 2010, founded the Cross Section and Mean Free Path to mammalians cell of Chinese hamster cells type V79. It was carried out by using two models (LQ, RCR). The upper value of $(\lambda)$ when $\quad \mathrm{LET}=11.0 \mathrm{KeV} / \mu \mathrm{m}$ is $\quad\left(\lambda_{\mathrm{LQ}}=16 \mathrm{~nm}\right.$, $\lambda_{\mathrm{RCR}}=2.72 \mathrm{~nm}$ ), The lower value of $(\lambda)$ when LET $=30.5 \mathrm{KeV} / \mu \mathrm{m}$ is $\left(\lambda_{\mathrm{LQ}}=0.608 \mathrm{~nm}\right.$, $\lambda_{\mathrm{RCR}}=0.3421 \mathrm{~nm}$ ) [8].The interaction cross section has investigated to become a good physical measure to study the size of the impact and to offer some idea about the physical mechanism in several fields of physics[6].

Table 1: - Survival curve factors for the inactivation of V79 cells with neutrons.

\begin{tabular}{|c|c|c|c|c|c|c|c|}
\hline $\begin{array}{c}\mathbf{E} \\
(\mathbf{M e V})\end{array}$ & $\begin{array}{c}\mathbf{L E T} \\
(\mathbf{K e V} / \boldsymbol{\mu m})\end{array}$ & $\begin{array}{c}\boldsymbol{\alpha}_{\mathbf{R C R}} \\
(\mathbf{G y})\end{array}$ & $\begin{array}{c}\boldsymbol{\alpha}_{\mathbf{L Q}} \\
(\mathbf{G y})\end{array}$ & $\begin{array}{c}\boldsymbol{\lambda}_{\mathbf{R C R}} \\
(\mathbf{n m})\end{array}$ & $\begin{array}{c}\boldsymbol{\sigma}_{\mathbf{R C R}} \\
\left(\boldsymbol{\mu m}^{2}\right)\end{array}$ & $\begin{array}{c}\boldsymbol{\lambda}_{\mathbf{L Q}} \\
(\mathbf{n m})\end{array}$ & $\begin{array}{c}\boldsymbol{\sigma}_{\mathbf{L Q}} \\
\left(\boldsymbol{\mu m}^{2}\right)\end{array}$ \\
\hline 0.22 & 55.094 & 1.4 & 0.7086 & 0.0242 & 12.3411 & 0.0478 & 6.2463 \\
\hline 0.34 & 51.234 & 6.694 & 1.446 & 0.0054 & 54.8737 & 0.0252 & 11.8535 \\
\hline 0.43 & 48.076 & 1.463 & 0.7479 & 0.0265 & 11.2536 & 0.0519 & 5.753 \\
\hline 0.583 & 42.754 & 1.465 & 1.3 & 0.0298 & 10.0215 & 0.0336 & 8.8928 \\
\hline 0.66 & 40.532 & 1.219 & 0.8376 & 0.0378 & 7.9054 & 0.055 & 5.4319 \\
\hline 1.0 & 32.193 & 0.9896 & 0.8935 & 0.0586 & 5.0973 & 0.0649 & 4.6023 \\
\hline 2.0 & 20.439 & 1.9 & 0.6833 & 0.048 & 6.2135 & 0.1336 & 2.2346 \\
\hline 6.0 & 9.0351 & 0.5069 & 0.2394 & 0.4074 & 0.7328 & 0.8625 & 0.3461 \\
\hline
\end{tabular}

Method:

Survival curves data for Neutron's irradiation of V79 cells were taken from literature. This was done for (8) eight survival curves ranging in energy between $0.22-6.0 \mathrm{MeV}[9,10]$. The survival curves obtained were fitted to the linear quadratic equation and the Repairable Conditionally Repairable Model (RCR) were written in Equation 1 [4], and Equation 2 [11], respectively.

$$
\begin{aligned}
S(D) & =\mathrm{e}^{\left(-\alpha D-\beta D^{2}\right)} \\
S(D) & =\mathrm{e}^{(-a D)}+\mathrm{bDe}^{(-c D)}
\end{aligned}
$$

Where $\mathrm{S}$ is called the surviving fraction, $\mathrm{D}$ represents dose in Gy unite, $\alpha$ and $\beta$ represents the contribution to cell destruction. Where $a, b$ and $\mathrm{c}$ fitting parameters. Table 1 shows the neutron energies utilized in this study and the $\alpha$-values which describe the initial Slopes the survival curve was utilized to compute the inactivation cross section which is defined by the Equation $3[12,13]$

$$
\sigma\left(\mu m^{2}\right)=\frac{0.16 \times L E T(\mathrm{KeV} / \mu \mathrm{m}) \times \alpha\left(\mathrm{Gy}^{-1}\right)}{\rho(\mathrm{g} / \mathrm{cm})^{3}}
$$

Where $\sigma$ describes the Inactivation cross section, LET represent Linear Energy Transfer and $\rho$ is the tissue density.

\section{Results and discussion:}

Figure (1-a) display the relation between the cross section and the neutrons energy, the relation was fluctuated at low energy below 1.0 $\mathrm{MeV}$, then $\sigma$ reduced by an increase the $\mathrm{E}(\mathrm{MeV})$. Figure (1-b) demonstration the relative between the inactivation cross-section $\sigma\left(\mu \mathrm{m}^{2}\right)$ and the mean free path $\lambda(\mathrm{nm})$ for the Linear Quadratic model (LQ). The behavior was exponentially for that relation thereby the inactivation cross-section declines to an increase the mean free path. The neutrons at low $\lambda$ are capable of create many free radicals 
and it have huge ionization density. The maximum value of cross section is about 12 $\mu \mathrm{m}^{2}$.the relation between the Linear Energy Transfer (LET) and the Neutrons Energy was exponentially curve shown in figure (1-c).
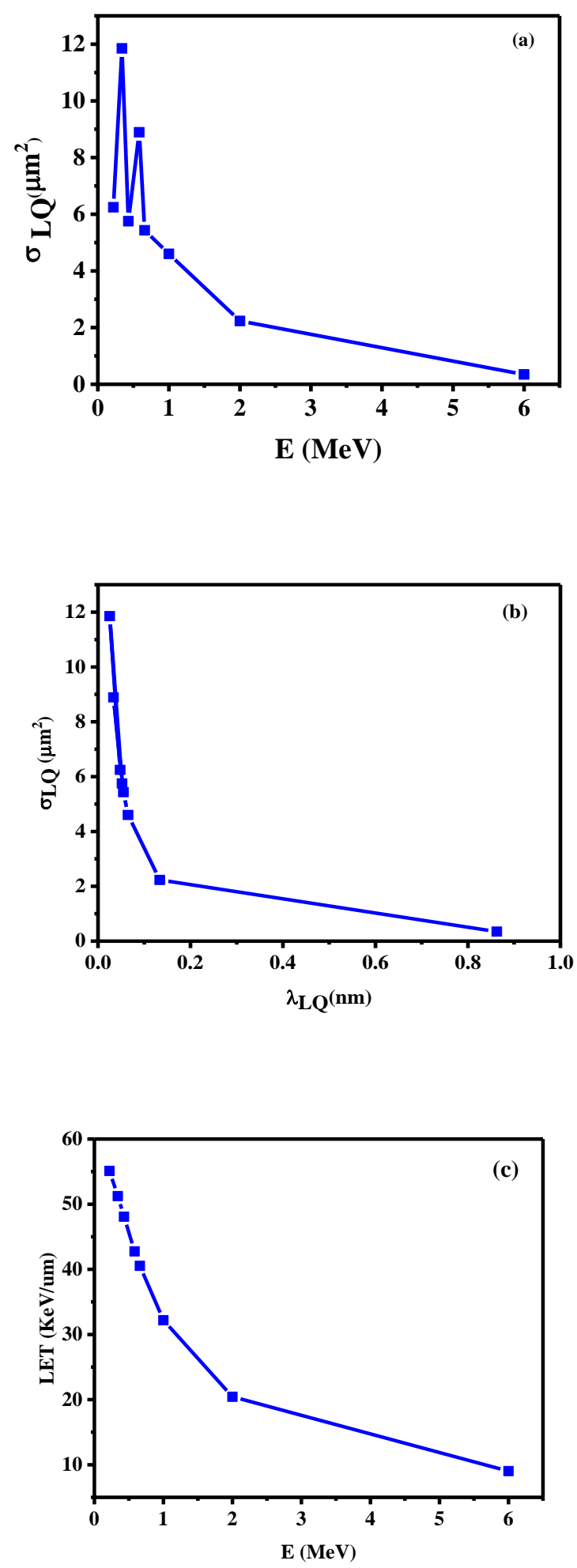

Figure (1): - explain (a) the relation between $\sigma$ and the neutrons energy, (b) the relation between the $\sigma-\lambda$ and (c) the relation between LET-E.

While for the Repairable Conditionally Repairable Model (RCR), the $\sigma$ have the highest value at $0.5 \mathrm{MeV}$ then it reduced with increase the neutrons energy as shown in figure (2-a). The relation between $\sigma-\lambda$ for RCR model followed the exponentially shape as shown in figure (2-b). Also, could be divided the region from the relation between the $\sigma$-E into four regions. At the energy range between (0.0-0.5) MeV the cross section increased but the $\sigma$ was reduced at $\lambda$ between $(0-0.3) \mathrm{nm}$ that can be attributed to the distant between two ionizations lower than the distant between DNA strands.

The inactivation cross section and mean free path for the neutrons Energy were plotted against the linear energy transfer LET which included in this study as shown in figure (3). the $\sigma_{\mathrm{LQ}}$ model is growing linearally behavior up to $40(\mathrm{KeV} / \mu \mathrm{m})$ then followed fluctuated behavior between (40-55) $\mathrm{KeV} / \mu \mathrm{m}$ as shown in figure (3a),also the $\sigma_{\mathrm{RCR}}$ is increasing semilinear shape up to $50(\mathrm{KeV} / \mu \mathrm{m})$ then increased to 51 after that decline near to $55(\mathrm{KeV} / \mu \mathrm{m})$ as shown in figure (3b). 

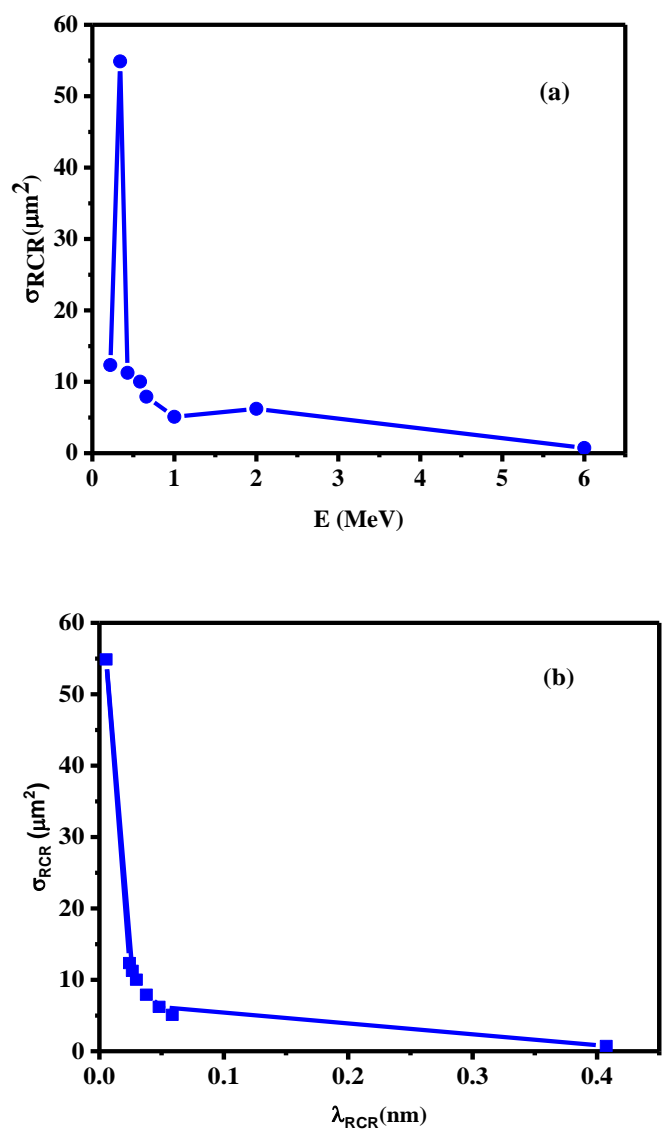

Figure (2): - (a) demonstrate the relation between the cross section and E, (b) display the relation between the Cross section and mean free path for RCR model.

The neutrons are capable to creation huge secondary particles due the collisions. The cross-section values relies on the ionization density on particle type. The highest value for $\sigma_{\mathrm{LQ}}$ is around $12\left(\mu \mathrm{m}^{2}\right)$, but for the $\sigma_{\mathrm{RCR}}$ is about $55\left(\mu \mathrm{m}^{2}\right)$. The $\sigma_{\mathrm{LQ}}$ value agree with Azooz and Meerkhan [7] and watt 1997 [1], however the $\sigma_{\mathrm{RCR}}$ value disagreement. While the $\lambda_{\mathrm{LQ}}$ and $\lambda_{\mathrm{RCR}}$ were reduced with increase the LET and cross section and followed the exponentially behavior as shown in figures ( $3 \mathrm{a}$, 3b).
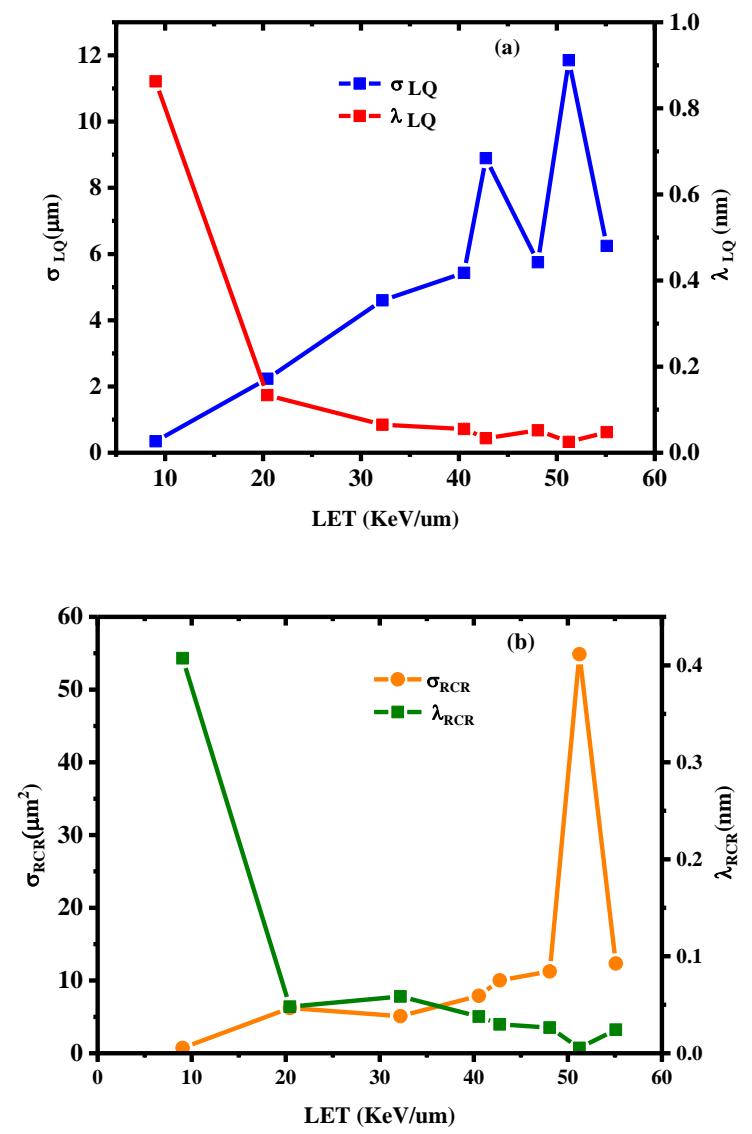

Figure (3):- demonstrate the relation between cross section and mean free path versus LET for the LQ and RCR models.

\section{Conclusions:}

Inactivation cross section consider a best factor to study the impact of radiation and damage mechanisms due to ionizing radiations. Observed from this study $\sigma_{\mathrm{LQ}}$ and $\sigma_{\mathrm{RCR}}$ of V79 cells irradiated by neutrons was increases nearly linearly at low LET and fluctuated at high LET and exponentially with Energy increasing $\lambda$ and this decrease is due to indirect action. The exponential figure is refered to the contact of secondary radicals with DNA to produce DSB, which are the main lead to cell damage. This procedure is defined as an indirect action method. The higher value of $(\lambda)$ when $\mathrm{LET}=9.0 \mathrm{KeV} / \mu \mathrm{m}$ is $\left(\lambda_{\mathrm{LQ}}=0.8625 \mathrm{~nm}\right.$, $\left.\lambda_{\mathrm{RCR}}=0.4074 \mathrm{~nm}\right)$, the minor value of $(\lambda)$ when LET $=51.32 \mathrm{KeV} / \mu \mathrm{m}$ is $\quad\left(\lambda_{\mathrm{LQ}}=0.0252 \mathrm{~nm}\right.$, $\left.\lambda_{\mathrm{RCR}}=0.0054 \mathrm{~nm}\right)$. 


\section{References}

1. Watt, D.E., 1997. A Unified System of Radiation Bio-Effectiveness and its Consequences in Practical Application. Radiat. Prot. Dosim., 70(1-4): pp. 529536.

2. Cember, H.T.E.J., Introduction to Health Physics. Fourth ed. 2008, the United States: McGraw-Hill Companies.

3. Simić, B., Nikolić, D., Stanković, K., Timotijević, L., and Stanković, S., 2013. Damage induced by neutron radiation on output characteristics of solar cells, photodiodes, and phototransistors. Int. J. Photoenergy.

4. Alpen, E.L., Radiation biophysics. 1997: Academic Press.

5. Watt, D., Al-Affan, I., Chen, C., and Thomas, G., 1985. Identification of biophysical mechanisms of damage by ionising radiation. Radiat. Prot. Dosim., 13(1-4): pp. 285-294.

6. Azooz, F.A. and Alkhalidy, T.J., 2007. Calculation of the Inactivation Cross Section of V79 Cells by Protons in Radiotherapy. Sultan Qaboos Univ. Med. J., 7(3): pp. 233.

7. Azooz, F.A. and Meerkhan, S.A., 2010. Phenomenological explanation of cell inactivation cross section in terms of direct and indirect action. Sultan Qaboos Univ. Med. J., 10(1): pp. 64.
8. Al-Ta'ii, H.M., 2010. Calculation of cross section area and Mean Free path for Interaction of protons with Mammalians cell of Chinese hamster cells (V79). Uruk, 3(1).

9. Hall, E.J., Novak, J.K., Kellerer, A.M., Rossi, H.H., Marino, S., and Goodman, L.J., 1975. RBE as a function of neutron energy: I. Experimental observations. Radiat. Res., 64(2): pp. 245-255.

10. Leenhouts, H. and Chadwick, K., 1973. A theoretical analysis of radiation sensitivity in cells following neutron irradiation. Biological Effects of Neutron Irradiation, IAEA-SM-179 (Vienna): pp. 151-163.

11. Lind, B., Persson, L., Edgren, M., Hedlöf, I., and Brahme, A., 2003. Repairableconditionally repairable damage model based on dual Poisson processes. Radiat. Res., 160(3): pp. 366-375.

12. Belli, M., Goodhead, D., Ianzini, F., Simone, G., and Tabocchini, M., 1992. Direct comparison of biological effectiveness of protons and alphaparticles of the same LET. II. Mutation induction at the HPRT locus in V79 cells. Int. J. Radiat. Biol., 61(5): pp. 625-629.

13. Watt, D.E. and Alkharam, A.S., 1994. Charged particle track structure parameters for application in Radiation Biology and Radiation Chemistry. Int. J. Quantum Chem., 52(S21): pp. 195207. 